\title{
Evolution and maintenance of cooperation via inheritance of neighborhood relationship
}

\author{
TAN ShaoLin ${ }^{1}$, LÜ JinHu ${ }^{1 *}$, YU XingHuo ${ }^{2} \&$ HILL David John ${ }^{3,4}$ \\ ${ }^{1}$ The Key Laboratory of System and Control, Institute of Systems Science, Academy of Mathematics and Systems Science, Chinese Academy of \\ Sciences, Beijing 100190, China; \\ ${ }^{2}$ School of Electrical and Computer Engineering, RMIT University, Melbourne, VIC 3001, Australia; \\ ${ }^{3}$ Department of Electrical and Electronic Engineering, The University of Hong Kong, Hong Kong, China; \\ ${ }^{4}$ School of Electrical and Information Engineering, University of Sydney, Sydney, NSW 2006, Australia
}

Received March 22, 2013; accepted May 21, 2013

\begin{abstract}
Cooperative behaviors are ubiquitous in nature and human society. It is very important to understand the internal mechanism of emergence and maintenance of cooperation. As we know now, the offsprings inherit not only the phenotype but also the neighborhood relationship of their parents. Some recent research results show that the interactions among individuals facilitate survival of cooperation through network reciprocity of clustering cooperators. This paper aims at introducing an inheritance mechanism of neighborhood relationship to explore the evolution of cooperation. In detail, a mathematical model is proposed to characterize the evolutionary process with the above inheritance mechanism. Theoretical analysis and numerical simulations indicate that high-level cooperation can emerge and be maintained for a wide variety of cost-to-benefit ratios, even if mutation happens during the evolving process.
\end{abstract}

complex networks, cooperation, evolutionary dynamics, population structure, coevolution

Citation: Tan S L, Lü J H, Yu X H, et al. Evolution and maintenance of cooperation via inheritance of spatial neighborhood. Chin Sci Bull, 2013, 58: 3491-3498, doi: 10.1007/s11434-013-5984-y

As we know now, cooperative behaviours are ubiquitous in nature and human society. Typical examples include mutual aid [1], communal group defence, coalition formation, and cooperative hunting $[2,3]$. It is well known that cooperation among individuals promotes common welfare of the whole population and helps individuals survive under some hostile environments. However, it may not naturally emerge and be maintained because it is costly to individuals and also can be easily exploited. On the contrary, selfish agents are normally favoured by natural selection without further mechanisms. Therefore, the inherent mechanism of cooperation has been a major puzzle of evolutionary biology for several decades [4-7].

To understand the puzzle of cooperation, many researchers have introduced various mathematical models to char-

\footnotetext{
*Corresponding author (email: jhlu@iss.ac.cn)
}

acterize its essence. In particular, the prisoner's dilemma game is one representative model [8-11]. In detail, a cooperator pays a cost $c$ to contribute a benefit $b$ to its partner while a defector pays no cost and contributes no benefits, where $b>c>0$. As we know now, the evolutionary dynamics of the prisoner's dilemma game indicates that defectors are favoured by selection while cooperators are eventually eliminated. In the evolutionary process of the prisoner's dilemma game, individuals engage in a pairwise game to gain their fitness. Not surprisingly, the fitness of defectors is always higher than that of cooperators in a well-mixed population. Therefore, the cooperation can hardly naturally emerge and be maintained.

To facilitate cooperation, various mechanisms have been proposed in evolutionary dynamics [12-21]. In particular, some recent results demonstrate that the interactions among individuals can facilitate survival of cooperation through 
network reciprocity of clustering cooperators [22-26]. Different from the traditional well-mixed population, individuals interact only with some neighbors in a structured population. In such a heterogeneous environment, cooperators could survive by forming cooperative clusters to resist the invasion and exploitation of defectors [27,28]. The above network effect is called network reciprocity.

Besides network reciprocity, the population structure also provides a necessary environment for coevolution. That is, in structured population, mobile individuals may migrate from one location to another to seek benefits and avoid disadvantages, which leads to the updating of population structure. As a result, mobile cooperators will always keep away from those defectors, which causes the elimination of defectors eventually. This is because the defectors can not take advantage of the cooperators any longer. In fact, some recent results validate the above mechanism and also find that cooperation can be preserved at high level in coevolutionary game dynamics [29-35].

Note that offsprings are usually reproduced nearby the habitat of their parents, and children will often inherit social, moral, political or religious beliefs just by proximity to their parents [36-38]. That is, an offspring inherits not only the traits but also the neighborhood relationship of its parents. Moreover, the above evolutionary mechanism can also lead to the coevolution of population structure and traits. Different from the coevolution caused by migration, individuals need not have the cognitive ability to distinguish defectors from cooperators in the coevolution caused by inheritance of neighbourhood relationship. This paper aims to introduce an inheritance mechanism of neighborhood relationship to further investigate the evolution of cooperation.

Following this line, this paper further investigates the effect of the above mechanism on the evolution of cooperation. In detail, we propose a minimalist stochastic model of natural selection with the inheritance mechanism of neighbourhood relationship. To begin with, all individuals are distributed in a given lattice. During the evolution, the eliminated individuals together with all their connected edges are deleted from the population, then offsprings are added in the population and also inhabit the neighborhood of their parents. Our theoretical analysis and numerical simulations show that the proposed inheritance mechanism of neighbourhood relationship can significantly promote the emergence of cooperation. More shockingly, we find that the cooperators can automatically cut off those mutated defectors to inhibit propagation of them in our evolutionary model.

The above inheritance mechanism of neighborhood relationship provides a simple and rigorous explanation for the widespread existance of cooperation behaviors. Different from some traditional cooperation mechanisms, the inheritance mechanism of neighborhood relationship is not based on the cognitive ability or social attributions of individuals. Moreover, the natural selection instead of social dynamics is used as the driving force of population evolution. Therefore, our developed model can be used to further investigate the evolution of cooperation in many biological species especially microbes, such as the bacterium Vibrio [3] and $M$. xanthus [36-38].

The remain paper is organized as follows. Section 1 introduces a coevolutionary model with inheritance mechanism of neighbourhood relationship. The dynamical behaviors of this model are further investigated in Section 2. Section 3 explores the inherent mechanism of the emergence of cooperation. Some concluding remarks are given in Section 4.

\section{The mathematical model}

Based on the evolutionary process of a structured population with cooperators and defectors, this section introduces a coevolutionary model with the inheritance mechanism of neighborhood relationship.

Consider a structured population. The population structure is characterized by a network, where nodes and edges represent individuals and the corresponding neighborhood relationships between individuals [39-45], respectively, as shown in Figure 1. Due to the limitation of sources, the number of individuals and interactions are normally assumed to be a constant during the whole evolutionary process.

At each step, each individual acquires its payoff $P$ by playing the prisoner's dilemma game described by

$\begin{array}{ccc} & \text { Cooperation } & \text { Defection } \\ \text { Cooperation } & b-c & -c \\ \text { Defection } & b & 0\end{array}$

with all individuals in its neighborhood. Assume that a cooperator has $k$ neighbors, where there are $i$ cooperators. Then the cooperator has a payoff $b i-c k$. Similarly, a defector with $j$ cooperative neighbors gets a payoff $b j$. To diminish the accumulation effect of payoff in large-degree nodes, the total payoff of an individual is often normalized by the number of its neighbors [46]. In detail, the fitness of an individual is defined by $F=1-w+w P$, where $P$ is the average payoff and $0 \leqslant w \leqslant 1$ is a parameter adjusting the selection intensity.

After game interaction, all individuals update their strategies. Here, the famous Moran process $[47,48]$ is used to characterize the updating process. In detail, an individual is randomly chosen to die firstly, then another individual is selected to reproduce an offspring with a probability proportional to its fitness. In the reproduction of offsprings, mutation happens at probability $u$. That is, the offspring inherits the parents' strategy with probability $1-u$, but with probability $u$, it mutates into a randomly strategy from cooperation to defection.

At the same time of updating the population traits, the population structure is also evolving in our model. In detail, 


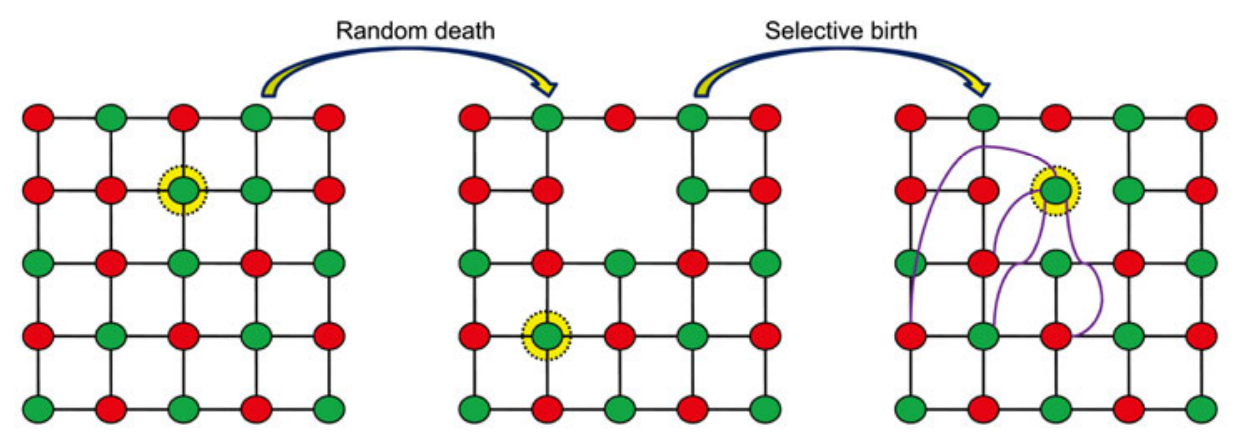

Figure 1 Sketch map of updating rule in the evolutionary process with inheritance mechanism of neighborhood relationship. Individuals occupy the vertices of a network. Blue and red nodes denote cooperators and defectors, respectively. The updating process is described by the following two steps. Step 1: Randomly choose an individual to die and then remove this node and its edges; Step 2: Select an individual with the probability proportional to fitness to reproduce an offspring, add a new node, and then connect this new node to its parent and the other three neighbors of its parent.

when an individual dies, the corresponding node and all edges of this node are removed from the network. And when an offspring is produced, a new node is added into the network to represent it. To characterize the inheritance mechanism of neighborhood relationship, this new node is connected to its parent and another $d-1$ nodes which are randomly chosen from the neighbors of its parent. Here, $d$ denotes the average degree of the network. Figure 1 shows the above updating process of population structure.

To summarize, the above model describes a complete evolutionary process of population with cooperators and defectors. It is worth mentioning that there are three typical characteristics in the above model. At first, the updating rule conforms to the three fundamental principles of natural evolution, i.e. reproduction, mutation and selection. Then, the individuals need not to possess any cognitive ability and social attributes, including punishment and reputation. Finally, the inheritance mechanism of neighborhood relationship is introduced in this model.

In this paper, assume that individuals occupy vertices of a two dimensional $n \times n$ lattice with periodic boundary at initial time [8]. Also, the cooperator and defector roles are randomly assigned to each individual.

\section{Evolutionary behaviors of cooperation}

\subsection{Effect of inheritance mechanism of neighborhood relationship}

This paper focuses on how cooperation emerges and is maintained during the evolutionary process. In detail, we are concerned mainly with the evolutionary behaviors of cooperation. Moreover, we further explore how cooperation behaves with the other parameters.

To begin with, we investigate the evolving process of cooperator frequency with the time. Figure 2 shows a typical evolutionary trace of cooperator frequency based on our model, where the population size, cost-to-benefit ratio $c / b$, selection strength $w$, and mutation rate $u$ are $10 \times 10,0.2,0.1$ and 0.01 , respectively. The evolutionary process starts from an unbiased state with average $50 \%$ cooperators in the population. Due to the exploitation of defectors, the proportion of cooperators decreases to around $40 \%$ in the earlier stage. In the traditional evolutionary models, this downward trend will keep on and then cooperators will be eventually eliminated out of the population, leading to the puzzle of cooperation. However, intriguingly here, such a scenario does not happen. Instead, the cooperators defeat the defectors and eventually take over most of or even all of the population at some time. Here, the mutation from cooperator to defector may cause the fluctuation of the frequency of cooperators.

We observe that there exists an impressive invasion at about the 30000th step, where the cooperators are on the verge of extinction. However, they can survive and eventually take back the whole population.

The typical evolutionary trace of cooperator frequency in Figure 2 indicates two fundamental laws of the above coevolutionary model in the following. Firstly, cooperation can emerge in our coevolutionary model, where the average cooperation level is 0.84 after 10000 generations. Secondly, cooperation can be maintained. In particular, cooperators can resist the invasion of mutated defectors and preserve a high cooperative level. To summarize, the above two phenomena are induced by the inheritance mechanism of neighborhood relationship. To validate the proposed inheritance mechanism, a control experiment is designed for comparison, where the strategies of population are updated while the structure of population is fixed. Figure 3 shows the average proportion of cooperators for each generation. As we expected, without the inheritance mechanism of neighborhood relationship, the cooperation level almost decreases to zero.

\subsection{Evolution of cooperation behaviors with parameters}

To further understand the effect of the inheritance mechanism of neighborhood relationship, it is necessary to explore the influence of other parameters on cooperation behaviors in both coevolutionary and control experiments. Figure 4 shows the average proportions of cooperators in the population 


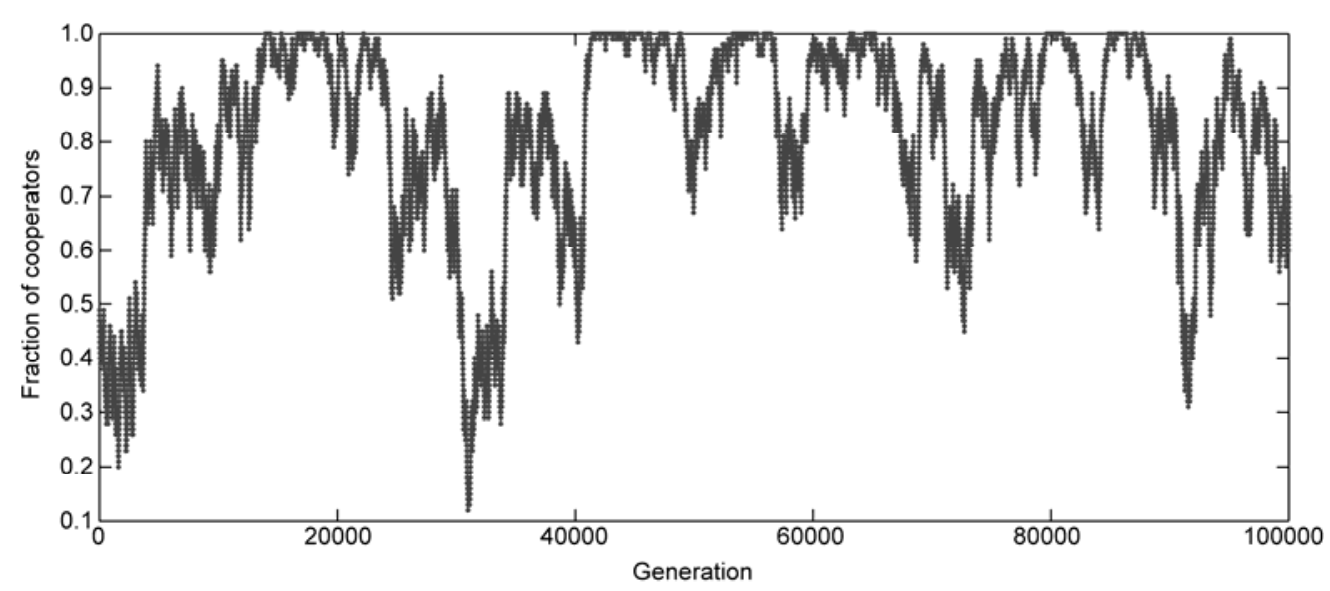

Figure 2 A typical evolutionary trace of cooperator frequency in the population. The average cooperation level is 0.84 after 10000 generations. The initial cooperators ratio is $50 \%$ and the individuals are randomly distributed on a lattice of size $10 \times 10$. The cost-to-benefit ratio, selection strength, and mutation rate are given by $c / b=0.2, w=0.1$, and $u=0.01$, respectively.

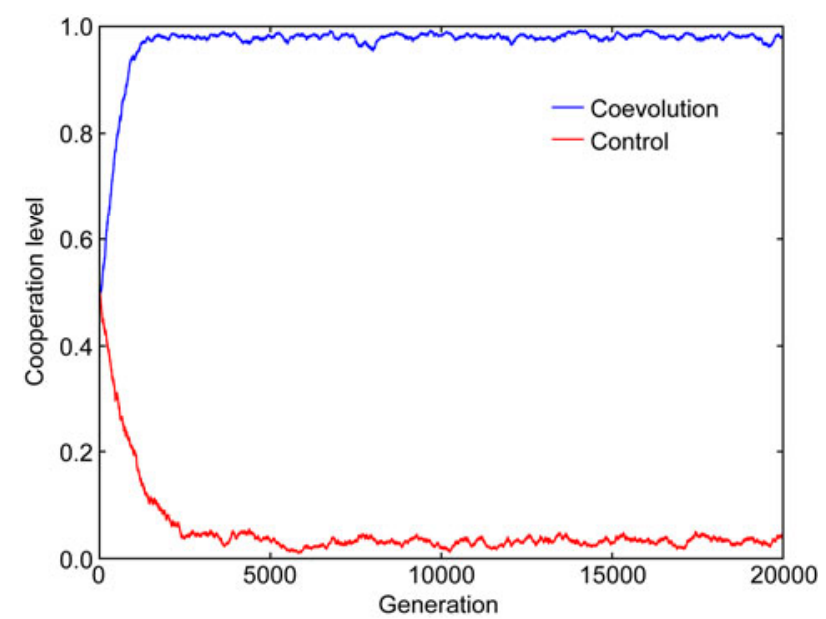

Figure 3 The relationship between average cooperation levels and generations, where the blue and red lines denote the cooperation level of coevolutionary model and the corresponding control experiment, respectively. In this figure, every data point is calculated by the average of 20 independent realizations. The initial cooperators ratio is $50 \%$ and the individuals are randomly distributed on a lattice of size $10 \times 10$. The cost-to-benefit ratio, selection strength, and mutation rate are given by $c / b=0.2, w=0.1$, and $u=0.01$, respectively.

as a function of the cost-to-benefit ratio $c / b$ in the equilibrium of evolutionary process.

From Figure 4, we have the following two interesting observations. At first, the cooperation level is very stable for a large scale of cost-to-benefit ratios, which indicates that the coevolutionary dynamics is insensitive to small values of $c / b$. Secondly, the cooperation favored threshold of cost-to-benefit ratio $c / b$ is extraordinarily high. Starting from an unbiased state, cooperation is called to be favored if the average cooperator frequency of evolutionary process at equilibrium is greater than 0.5 . Figure 4 indicates that cooperation is favored until $c / b$ exceeds 0.93 in the coevolutionary model. Note that cooperation is favored for $c / b<$

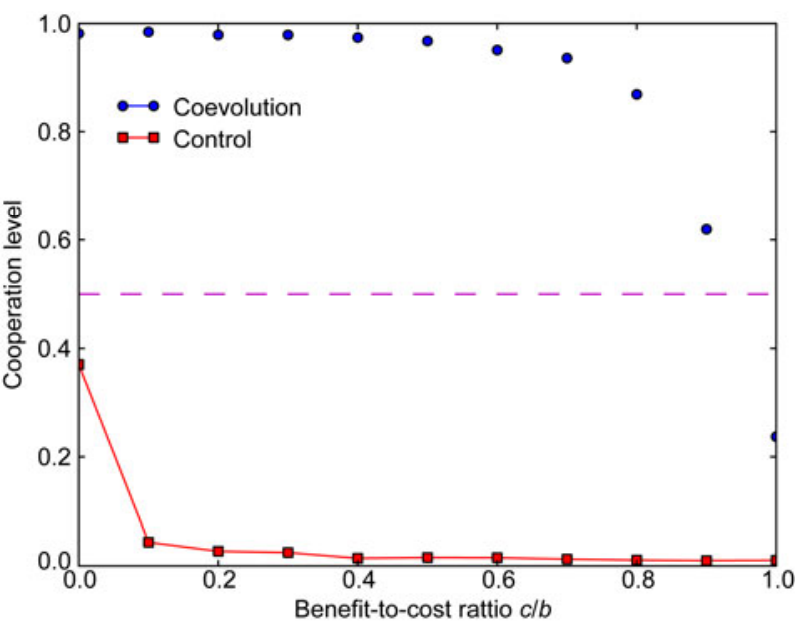

Figure 4 The relationship between average cooperation level and cost-tobenefit ratio $c / b$. The initial cooperators ratio is $50 \%$ and the individuals are randomly distributed on a lattice of size $8 \times 8$. The selection strength and the mutation rate are given by $w=0.5$ and $u=0.01$, respectively. Here, each data point is obtained by averaging the 20 independent realizations. And the cooperation level is calculated by averaging the cooperator frequency between 10000-th to 20000-th generations.

0.25 [22] in death-birth process on periodic lattice. Similarly, the threshold is 0.464 [49] in evolution of cooperation by phenotype similarity. Obviously, compared with the above two traditional mechanisms, the proposed inheritance mechanism of neighborhood relationship is much more effective for enhancing cooperation. In particular, under the above coevolutionary dynamics, cooperation can be favored even in some hostile conditions, such as the larger value $c / b$. However, without this coevolutionary mechanism, defectors dominate the population in the control experiment as shown in Figure 4.

Figure 5 (a) shows the relationship between average cooperation level and selection strength $w$. When there does not exist selection, i.e. $w=0$, the evolutionary behaviors of 
cooperation are completely characterized by neutral drifts [50]. In this case, the cooperator frequency will remain unchanged from generation to generation in the statistical sense. Thus, for $w=0$, the cooperation level is almost the same as the initial cooperator frequency, i.e. 0.5 , for both coevolutionary and control models. For $w>0$, the payoffs derived from the pairwise game contribute to and cause differences in the fitness of individuals. Such differences benefit to the survival of cooperators in the coevolutionary model. In fact, the cooperation level increases with the selection strength. However, in the control experiment, the cooperation level decreases with $w$. These observations indicate that the selection will favor cooperators rather than defectors under the inheritance mechanism of neighborhood relationship in the evolutionary process of population.

To know whether cooperation can be maintained, it is necessary to explore the effect of mutation in the evolutionary process. According to the literature, most known results focus on the emergence of cooperation, instead of the maintenance of cooperation. In our coevolutionary model, the cooperators can take over the whole population if there does not exist mutation as shown in Figure 5(b). However, the cooperation level decreases rapidly if there exists mutation. That is, mutation makes survival of cooperation much harder. This is because the cooperators face invasion not only from outside defectors but also from inner mutated defectors if there exists mutation. Though, for a small mutation rate, such as $u<0.2$, cooperation can still thrive and be promoted in some level. Therefore, cooperation can not only emerge but also be maintained in our coevolutionary model.

In the coevolutionary dynamics, cooperation is robust with respect to the population size and initial structures. In detail, the evolutionary features will remain almost unchanged for different initial structures, such as regular lattice, random graphs, and random geometric graphs, and population sizes. For the long-term view, the cooperation level is maintained almost the same as shown in Figure 6.

\section{Emerging mechanism analysis of cooperation}

The above results have shown the positive effect of the inheritance mechanism of neighborhood relationship on enhancing cooperation. However, it is still unclear how this mechanism works. In the following, we further explore the emerging mechanism of cooperation.

In detail, the invasion process of a mutated defector into a population of cooperators is used to illustrate how defectors are finally eliminated out of the population. The analysis is mainly based on a fundamental property of Moran process, i.e. "survival of the fittest". It means that the individuals with low fitness will be eventually eliminated out of the population and those with high fitness will survive $[47,48]$.

As outlined in Figure 7, at the beginning of the invasion, the defector is surrounded by cooperators and thus has the largest fitness. Under the Moran process, this defector is very likely to be selected to reproduce offsprings. So in this stage, the frequency of defectors increases and that of cooperators decreases, respectively. Due to the inheritance mechanism of neighborhood relationship, these defectors inhabit nearby locations in the space as shown in stage 1 of Figure 7. With the increase of defectors, the fitness of the neighboring cooperators around these defectors decreases rapidly. Thus, these cooperators will be eventually eliminated out of the
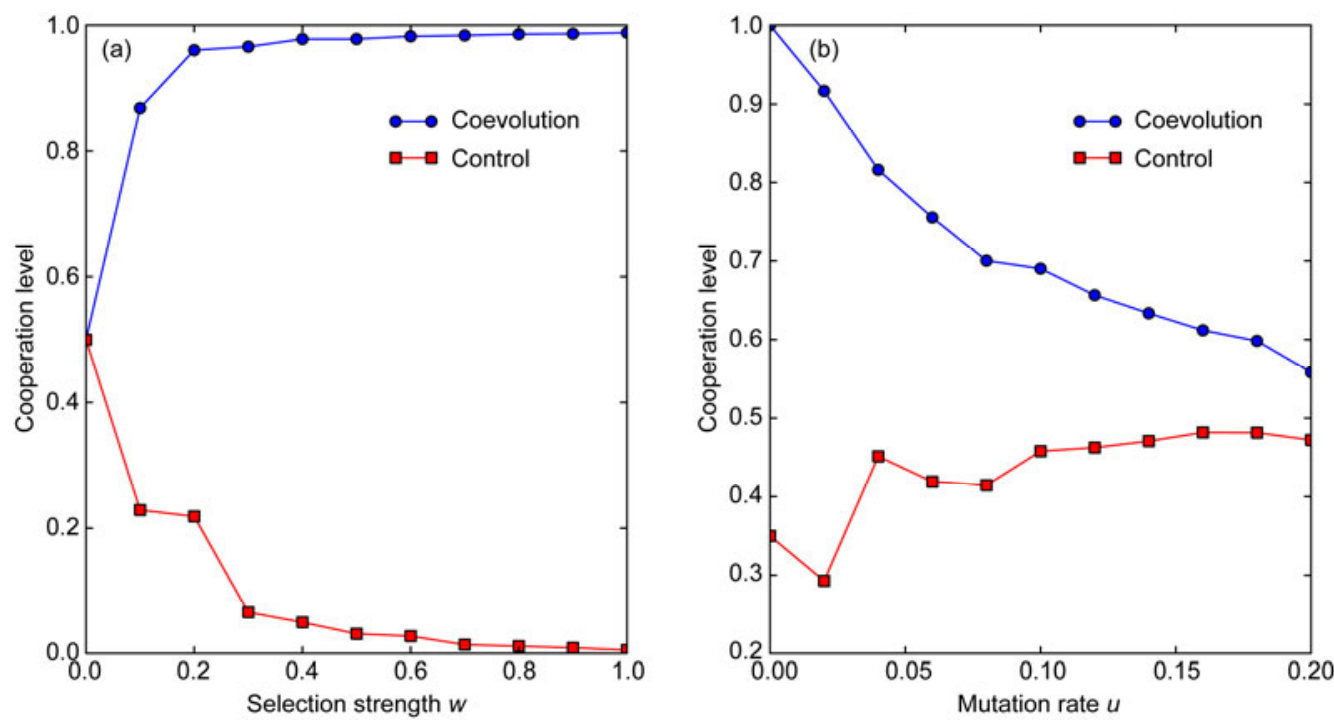

Figure 5 (a) The relationship between average cooperation level and selection strength $w$ with $u=0.01$; (b) The relationship between average cooperation level and mutation rate $u$ with $w=0.1$. The red square line denotes the control experiment with fixed structure. The initial cooperators ratio is $50 \%$ and the individuals are randomly distributed on a lattice of size $8 \times 8$. The cost-to-benefit ratio is given by $c / b=0.2$. Here, each data point is obtained by averaging the 20 independent realizations. And the cooperation level is calculated by averaging the cooperator frequency between 10000 -th to 20000 -th generations. 


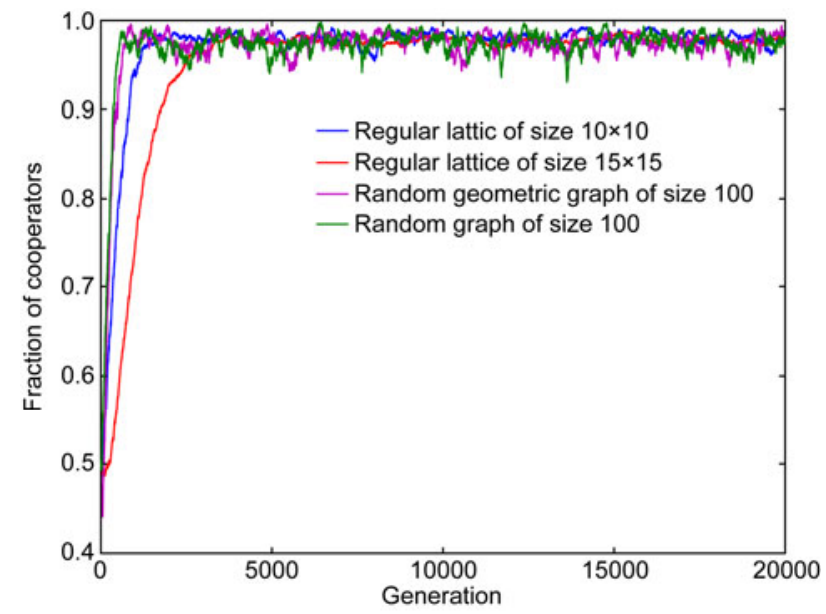

Figure 6 The effect of initial population structure and population size on the evolutionary behaviors of cooperation. Each data point is obtained by averaging the 20 independent realizations. Different colored lines denote the cooperation levels on different initial structures. The cost-to-benefit ratio, selection strength, and mutation rate are given by $c / b=0.2, w=0.1$, and $u=0.01$, respectively.

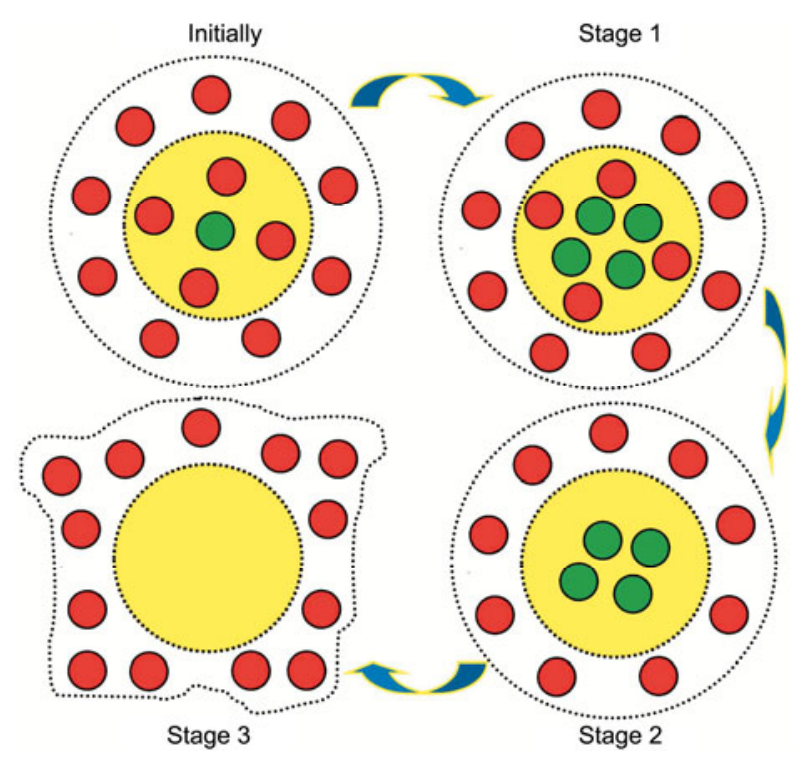

Figure 7 Illustration of an invasion process of mutated defector. Individuals are distributed in a given space, where red and green nodes denote the cooperators and defectors, respectively. To begin with, a cooperator mutates into a defector. The defector with the highest fitness survives and reproduces many offsprings as shown in Stage 1. At the same time, due to the exploitation of defectors, the neighboring cooperators receive a low fitness, and thus eventually die out, as shown in Stage 2. As a result, the defectors get a very low fitness without cooperators in their neighborhood. Therefore, the defectors are eventually eliminated out of the population. And the population returns to a state of complete cooperation.

population as shown in stage 2 of Figure 7. Finally, there does not exist any cooperator in the neighborhood of defectors after these cooperators are eliminated. As a result, the fitness of these defectors decreases to the lowest. Then all defectors are eventually eliminated and the population is taken over by cooperators again as shown in stage 3 of Figure 7 .
To summarize, with the inheritance mechanism of neighborhood relationship, the cooperators can automatically cut off the neighboring cooperators around the defectors to isolate the mutated defectors and then eliminate them. Indeed, this explains why cooperators emerge and can be maintained in a high level in our coevolutionary dynamics.

It is well known that a necessary and essential route towards evolution of cooperation is assortment between individuals of the same type [51]. Cooperative mechanisms, such as kin selection [13], multi-level selection [21], punishment [34], and network reciprocity [12], ultimately, all lead to assortment of cooperative interactions.

In fact, the inheritance mechanism of neighborhood relationship also leads to the assortment of cooperative interactions. Under the Moran process, individuals living in a cooperative environment acquire a high fitness and then are very likely to be selected to reproduce offsprings. With the inheritance mechanism of neighborhood relationship, these offsprings locate in the same cooperative neighborhood, which leads to a positive feedback of interactions between cooperators. In contrast, due to elimination of the neighboring cooperators, the number of defector-cooperator interactions gradually reduces without mutation. Therefore, in our coevolutionary model, the strength of cooperator-cooperator assortment is significantly high. In this case, cooperators receive much more net fitness benefits from their neighbors. This is why cooperation can survive and be maintained at a high level even in some hostile conditions, such as high cost-to-benefit ratio and mutation rate.

The essential law of cooperation lies in that it provides either a direct or an indirect benefit to cooperators so that the fitness of cooperators overwhelms that of defectors [52-56]. On the one hand, we have shown that the inheritance mechanism of neighborhood relationship enhances the direct benefits of cooperators. On the other hand, it can also lead to the maximal inclusive fitness of individuals. Indeed, in our coevolutionary model, offsprings locate at the neighborhood of their parents, which keeps relatives together. Therefore, interacting cooperators are most likely to be relatives [57-59]. Such interactions not only bring direct fitness to the individuals involved but also enforce indirect inclusive fitness.

As we know now, the coevolution of structure and strategy is a fundamental mechanism for the evolution of cooperation. However, the proposed coevolution mechanism in this paper differs from the traditional coevolution mechanisms. Among these results, migration, punishment, or reputation are often regarded as the basic causes of structure updating [31-33,35]. Therefore, the individuals are implicitly required to have some social attributions and cognitive ability to detect the attributions of their neighbors. In contrast, in our model, the updating of population structure is based on the inheritance mechanism of neighborhood relationship. And the individuals do not need any additional 
requirements $[60,61]$.

Moreover, some other works update their population strategy with social dynamics, such as imitation, personal learning, and comparison. Here, the structure of population refers to social interactions [53-55]. The above results can only explain the formation of cooperation in humans. Different from the above models, our model explicitly adopts natural selection as the driving force of evolution. It aims to provide a feasible explanation for the mechanism of how cooperation emerges and can be maintained in natural evolution.

It should be especially pointed out that the inheritance mechanism of neighborhood relationship takes full advantage of natural selection andnetwork structure of the population. In fact, it can be regarded as the complement of network reciprocity on static structures. Note that network reciprocity works only under some favorable conditions, such as the low cost-to-benefit ratio [56]. However, it may not work well if the mutation rate exceeds some given threshold. In this case, the cluster of cooperators will break down if a mutated defector happens within this cluster. In particular, our coevolutionary mechanism can compensate for the above two disadvantages. Indeed, it can favor cooperators even in some hostile environment. Also, it is robust with respect to the variations of selection strength and mutation rate.

\section{Conclusion}

This paper has further investigated the effect of the inheritance mechanism of neighborhood relationship on the emergence of cooperation in structured population. For the inheritance mechanism of neighborhood relationship, the offsprings inherit not only the traits but also part of neighborhood relationships from their parents. Our research results show that selection favors cooperators over defectors under the above evolutionary mechanism. In fact, this above result holds for a wide variety of conditions, even for extraordinary high temptation of defection. Moreover, even the mutation happens, cooperation can still survive and be maintained at a high level. In particular, we have illustrated the emerging mechanism of cooperation by analyzing the invasion pattern of a single defector. Intriguingly, we also find that the population can automatically cut off defectors to inhibit the expanding of these defectors under the joint effect of natural selection and the inheritance mechanism of neighborhood relationship.

This work was supported by the National Natural Science Foundation of China (61025017, 11072254, and 61203148).

1 Kropotkin P. Mutual Aid: A Factor of Evolution. New York: Double Day, 1902
2 Kappeler P M, Schaik C P van. Cooperation in Primates and Humans. Berlin: Springer, 2005

3 Morlon H. Microbial cooperative warfare. Science, 2012, 337: 1184-1185

4 Chen Y, Lü J, Yu X. Robust consensus of multi-agent systems with time-varying delays in noisy environment. Sci China Tech Sci, 2011, 54: 2014-2023

5 Dugatkin L A. Cooperation Among Animals: An Evolutionary Perspective. New York: Oxford University Press, 1997

6 Johnson D D P, Stopka P, Knights S. Sociology-the puzzle of human cooperation. Nature, 2003, 421: 911-912

7 Fehr E, Fischbacher U. The nature of human altruism. Nature, 2003, 425: 785-791

8 Nowak M A, May R M. Evolutionary games and spatial chaos. Nature, 1992, 359: 826-829

9 Hofbauer J, Sigmund K. Evolutionary Games and Population Dynamics. Cambridge: Cambridge University Press, 1998

10 Nowak M A. Evolutionary Dynamics: Exploring The Equation of Life. Cambridge: Harvard University Press, 2006

11 Levin S A. Games, Groups, and The Global Good. Berlin: Springer, 2009

12 Nowak M A. Five rules for the evolution of cooperation. Science, 2006, 314: 1560-1563

13 Hamilton W D. Genetical evolution of social behavior. J Theor Biol, 1964, 7: 1-52

14 Trivers R L. The evolution of reciprocal altruism. Q Rev Biol, 1971, 46: $35-57$

15 Nowak M A, Sigmund K. Evolution of indirect reciprocity. Nature, 2005, 437: 1291-1298

16 Wilson D S A. Theory of group selection. Proc Natl Acad Sci USA, 1975, 72: 143-146

17 Li M, Song H, Zhang L, et al. Maintenance of cooperation in a public goods game: A new decision-making criterion with incomplete information. Chin Sci Bull, 2012, 57: 579-583

18 Wang X, Han J, Han H. Special agents can promote cooperation in the population. PLoS One, 2011, 6: e29182

19 Yang H, Wang B. Universal role of migration in the evolution of cooperation. Chin Sci Bull, 2011, 56: 3693-3696

20 Cremer J, Melbinger A, Frey E. Growth dynamics and the evolution of cooperation in microbial populations. Sci Rep, 2012, 2: 10.1038/ srep00281

21 Traulsen A, Nowak M A. Evolution of cooperation by multilevel selection. Proc Natl Acad Sci USA, 2006, 103: 10952-10955

22 Ohtsuki H, Hauert C, Lieberman E, et al. A simple rule for the evolution of cooperation on graphs and social networks. Nature, 2006, 441: 502-505

23 Santos F C, Pacheco J M, Lenaerts T. Evolutionary dynamics of social dilemmas in structured heterogeneous populations. Proc Natl Acad Sci USA, 2006, 103: 3490-3494

24 Szabo G, Fath G. Evolutionary games on graphs. Phys Rep, 2007, 446: 97-216

$25 \mathrm{Xu} \mathrm{Y,} \mathrm{Wu} \mathrm{Q,} \mathrm{Wang} \mathrm{J,} \mathrm{et} \mathrm{al.} \mathrm{Social} \mathrm{welfare} \mathrm{maximization} \mathrm{for} \mathrm{SRSNs}$ using bio-inspired community cooperation mechanism. Chin Sci Bull, 2012, 57: 125-131

26 Pinheiro F L, Pacheco J M, Santos F C. From local to global dilemmas in social networks. PLoS One, 2012, 7: e0032114

27 Voelkl B, Kasper C. Social structure of primate interaction networks facilitates the emergence of cooperation. Biol Lett, 2009, 5: 462-464

28 Lehman C L, Keller L, Sumpter D J T. The evolution of helping and harming on graphs: The return of the inclusive fitness effect. J Evolution Biol, 2007, 20: 2284-2295

29 Wu B, Zhou D, Fu F, et al. Evolution of cooperation on stochastic dynamical networks. PLoS One, 2010, 5: e11187

30 Perc M, Szolnoki A. Coevolutionary games-a mini review. Biosystems, 2010, 99: 109-125

31 Vainstein M H, Silva A T C, Arenzon J J. Does mobility decrease cooperation. J Theor Biol, 2007, 244: 722-728

32 Helbing D, Yu W. Migration as a mechanism to promote cooperation. Adv Complex Syst, 2008, 11: 641-652 
33 Rand D G, Arbesman S, Christakis N A. Dynamic social networks promote cooperation in experiments with humans. Proc Natl Acad Sci USA, 2011, 108: 19193-19198

34 Wang J, Suri S, Watts D J. Cooperation and assortativity with dynamic partner updating. Proc Natl Acad Sci USA, 2012, 109: 1436314368

35 Cong R, Wu B, Qiu Y, et al. Evolution of cooperation driven by reputation-based migration. PLoS One, 2012, 7: e35776

36 Velicer G J, Yu Y N. Evolution of novel cooperative swarming in the bacterium Myxococcus xanthus. Nature, 2003, 425: 75-78

37 Rainey P B, Rainey K. Evolution of cooperation and conflict in experimental bacterial populations. Nature, 2003, 425: 72-74

38 Griffin A S, West S A, Buckling A. Cooperation and competition in pathogenic bacteria. Nature, 2004, 430: 1024-1027

39 Lieberman E, Hauert C, Nowak M A. Evolutionary dynamics on graphs. Nature, 2005, 433: 312-316

40 Tan S, Lü J, Setti G. An analysis of fixation probability of a mutant on a class of weighted networks under neutral selection. In: Proc the 37th Annual Conf IEEE Industr Electr Soc. New Jersey: 2011. 40244028

41 Tan S, Lü J, Yu X, et al. Exploring evolutionary dynamics in a class of structured populations. In: Proc the 2012 IEEE Int Symp Circuits Syst. Seoul: 2012. 169-172

42 Tan S, Lü J, Yu X, et al. Monotonicity of fixation probability of evolutionary dynamics on complex networks. In: Proc the 38th Annual Conf IEEE Industr Electr Soc. Montreal: 2012. 2325-2329

43 Lü J, Chen G. A time-varying complex dynamical network model and its controlled synchronization criteria. IEEE Trans Automat Contr, 2005, 50: 841-846

44 Lü J, Yu X, Chen G, et al. Characterizing the synchronizability of small-world dynamical networks. IEEE Trans Circuits Syst I, 2004, 51: 787-796

45 Wang P, Lü J, Ogorzalek M J. Global relative parameter sensitivities of the feed-forward loops in genetic networks. Neurocomputing, 2012, 78: 155-165

46 Zhang J, Chen X, Zhang C, et al. Elimination mechanism promotes cooperation in coevolutionary prisoner's dilemma games. Physica A,
2010, 389: 4081-4086

47 Moran P A P. The Statistical Processes in Genetics. Oxford: Clarendon Press, 1962

48 Ewens W J. Mathematical Population Genetics. New York: Springer, 2004

49 Antal T, Ohtsuki H, Wakeley J, et al. Evolution of cooperation by phenotypic similarity. Proc Natl Acad Sci USA, 2009, 106: 8597-8600

50 Kimura M. The Neutral Theory of Molecular Evolution. Cambridge: Cambridge University Press, 1983

51 Fletcher J A, Doebeli M. A simple and general explanation for the evolution of altruism. Proc R Soc B, 2009, 276: 13-19

52 West S A, Griffin A S, Gardner A. Social semantics: altruism, cooperation, mutualism, strong reciprocity and group selection. J Evol Biol, 2007, 20: 415-432

53 Santos F C, Pacheco J M. Scale-free networks provide a unifying framework for the emergence of cooperation. Phys Rev Lett, 2005, 95: 098104

54 Traulsen A, Semmann D, Sommerfeld R D, et al. Human strategy updating in evolutionary games. Phys Rev Lett, 2010, 107: 29622966

55 McNally L, Brown S P, Jackson A L. Cooperation and the evolution of intelligence. Proc Royal Soc B, 2012, 279: 3027-3034

56 Langer P, Nowak M A, Hauert C. Spatial invasion of cooperation. J Theor Biol, 2008, 250: 634-641

57 Chen Y, Lü J, Lin Z. Consensus of discrete-time multi-agent systems with transmission nonlinearity. Automatica, 2013, 49: 1768-1775

58 Zhu J, Lü J, Yu X. Flocking of multi-agent non-holonomic systems with proximity graphs. IEEE Trans Circuits Syst I, 2013, 60: 199210

59 Chen Y, Lü J, Han F, et al. On the cluster consensus of discrete-time multi-agent systems. Syst Contr Lett, 2011, 60: 517-523

60 Lü J, Zhou T, Chen G, et al. Local bifurcations of the Chen system. Int J Bifurcation Chaos, 2002, 12: 2257-2270

61 Yu S, Lü J, Yu X, et al. Design and implementation of grid multiwing hyperchaotic Lorenz system family via switching control and constructing super-heteroclinic loops. IEEE Trans Circuits Syst I, 2012, 59: 1015-1028

Open Access This article is distributed under the terms of the Creative Commons Attribution License which permits any use, distribution, and reproduction in any medium, provided the original author(s) and source are credited. 